\title{
ORIGINAL ARTICLE \\ PROVINCIAL DIFFERENCES IN INFANT DEATHS IN SOUTH AFRICA - AN EFFECT OF ANTIRETROVIRAL INTERVENTIONS?
}

\author{
A Boulle, ${ }^{1} \mathrm{MB} C h B, P h D$ \\ M L Thompson, ${ }^{1,2} P h D$ \\ R Laubscher, ${ }^{3} B C o m$ \\ L F Johnson, ${ }^{1} P h D, A I A$ \\ R Sayed, ${ }^{1} M S c$ \\ L L Brody, ${ }^{1} M P H$ \\ B Draper, ${ }^{4} \mathrm{MB} C h B, \mathrm{MMed}$ \\ M F Cotton, ${ }^{5} \mathrm{MMed}, \mathrm{PhD}$ \\ F Abdullah, ${ }^{6} \mathrm{MB} C h B, B S c$ (Hons), FCPMH (SA) \\ J E Myers, ${ }^{1} B S c, M B C h B, D T M \& H, M D$ \\ D E Bourne, ${ }^{1} B S c$, BPhil \\ ${ }^{1}$ School of Public Health and Family Medicine, Faculty of Health Sciences, University of Cape Town \\ ${ }^{2}$ Department of Biostatistics, University of Washington, Seattle, USA \\ ${ }^{3}$ Biostatistics Unit, South African Medical Research Council, Cape Town \\ ${ }^{4}$ Knowledge Translation Unit, Lung Institute, University of Cape Town \\ ${ }^{5}$ Department of Paediatrics and Child Health, Stellenbosch University, Tygerberg, $W$ Cape \\ ${ }^{6}$ The Global Fund to Fight AIDS, Tuberculosis and Malaria, Geneva, Switzerland
}

Objective. It has previously been demonstrated that a peak in registered infant deaths, at 2 - 3 months of age at death, developed between 1997 and 2002 in South Africa, alongside the evolving HIV epidemic. The objective of this analysis was to explore the age distribution of post-neonatal infant deaths in South Africa by province, and relate the observed distributions to HIV and intervention characteristics.

Design. Ecological study based on registered infant deaths and published HIV and intervention characteristics.

Methods. Numbers of registered infant deaths beyond 1 month of age at death were plotted by year of death, province of South Africa and age at death in months, for the years 1997 - 2007.

Results. The total number of registered deaths in infants aged 1 - 11 months increased from 15404 in 1997 to 34479 in 2006. Eight of the 9 provinces experienced an annual peak in registered infant deaths at $2-3$ months of age between 1997 and 2007. This peak in mortality was not observed in the Western Cape. In 7 of 9 provinces registered post-neonatal infant deaths did not rise markedly in 2007 compared with 2005.

Conclusions. We identified a single province out of 9 South African provinces in which a peak in early infant deaths at age 2 - 3 months did not occur during the period 1997 - 2007. This was the province with the earliest and highest coverage of antiretroviral interventions from 1999 onwards. It is possible that these interventions have averted the greater increase in early infant deaths seen in the rest of South Africa over this period.

The response to HIV in sub-Saharan Africa (SSA) in the past decade has resulted in one of the largest and most dramatic health interventions in recent history, ${ }^{1}$ with the provision of antiretroviral therapy (ART) both as treatment to infected adults and children and to prevent vertical transmission of HIV from mother to child.

Where monitoring systems have been able to track outcomes of large-scale ART programmes in SSA, they appear to be effective. ${ }^{2,3}$ However, outside of small demographic surveillance sites ${ }^{4-6}$ weak vital registration systems limit our ability to demonstrate the populationlevel impact of these interventions. South Africa is unique in having a high burden of HIV disease together with one of the most complete vital registration systems on the continent, in which $80 \%$ of deaths are currently estimated to be registered. ${ }^{7}$

We have previously demonstrated in South Africa at a national level that infant deaths (at ages 1 - 11 months) increased over the period $1997-2002,{ }^{8}$ with the greatest increase at 2 - 3 months of age. This peak in early 
infant deaths could not be ascribed solely to improving registration of deaths over time, and was consistent with cohort studies of HIV-infected infants. ${ }^{9-10}$

In this present analysis we describe post-neonatal infant mortality by age in months across the 9 provinces in South Africa from 1997 to 2007, and reflect on the relationship between the early peak in infant deaths and province-specific HIV epidemic and intervention characteristics.

\section{SETTING}

Antenatal HIV-1 seroprovalence had reached $17.0 \%$ in pregnant women in South Africa by 1996, rose steadily to $30.2 \%$ by 2005 , and has subsequently remained around this level. ${ }^{11}$ In 2005, the HIV seroprevalence varied between provinces from $15.7 \%$ in the Western Cape to $39.1 \%$ in KwaZulu-Natal (Table I). There have also been large inter-provincial variations in both timing and coverage of antiretroviral interventions in South Africa. ${ }^{12}$

\section{METHODS}

We obtained South African infant mortality counts by age at death for the period 1997 - 2007 from Statistics South Africa. Infant deaths under 1 month were excluded from analysis because of the potential misclassification of live and stillbirths. Absolute counts of infant deaths were considered by province, year and age at death in months, starting at 1 month of age. For graphical presentation purposes, counts were averaged across the periods 1997 - 1999, 2000 - 2001, 2002 - 2003, 2004 2005 and 2006 - 2007. All analyses were conducted using Stata statistical software v11.0 (Stata-Corp Inc, College Station, Texas). The study was approved by the University of Cape Town Research Ethics Committee.

\section{RESULTS}

The total number of registered deaths in infants aged 1 - 11 months increased from 15404 in 1997 to 34479 in 2006 (32 828 in 2007). Eight of the 9 provinces experienced an annual peak in registered infant deaths at 2 - 3 months of age between 1997 and 2007 (Fig. 1). This peak in mortality was not observed in the Western Cape. In 7 of 9 provinces registered post-neonatal infant deaths did not rise markedly in 2007 compared with 2005 (Table I). Expressed as a ratio to deaths at 11 months of age (which compensates for increasing registration over time), this peak was still present in 2006 - 2007 in the 8 provinces where it was observed, but had declined in 5 compared with the ratios seen in previous calendar periods.

\section{DISCUSSION}

Our previous study demonstrated, at a national level for the period 1997 - 2002, a year-on-year increase in infant mortality at each age of death (1-11 months), with the greatest increase at 2 - 3 months. ${ }^{8}$ This current analysis by province for the period 1997 - 2007 demonstrates the continued presence of a peak in infant deaths at 2 - 3 months of age in the context of the evolving HIV epidemic in South Africa, with reported mortality stabilising in most
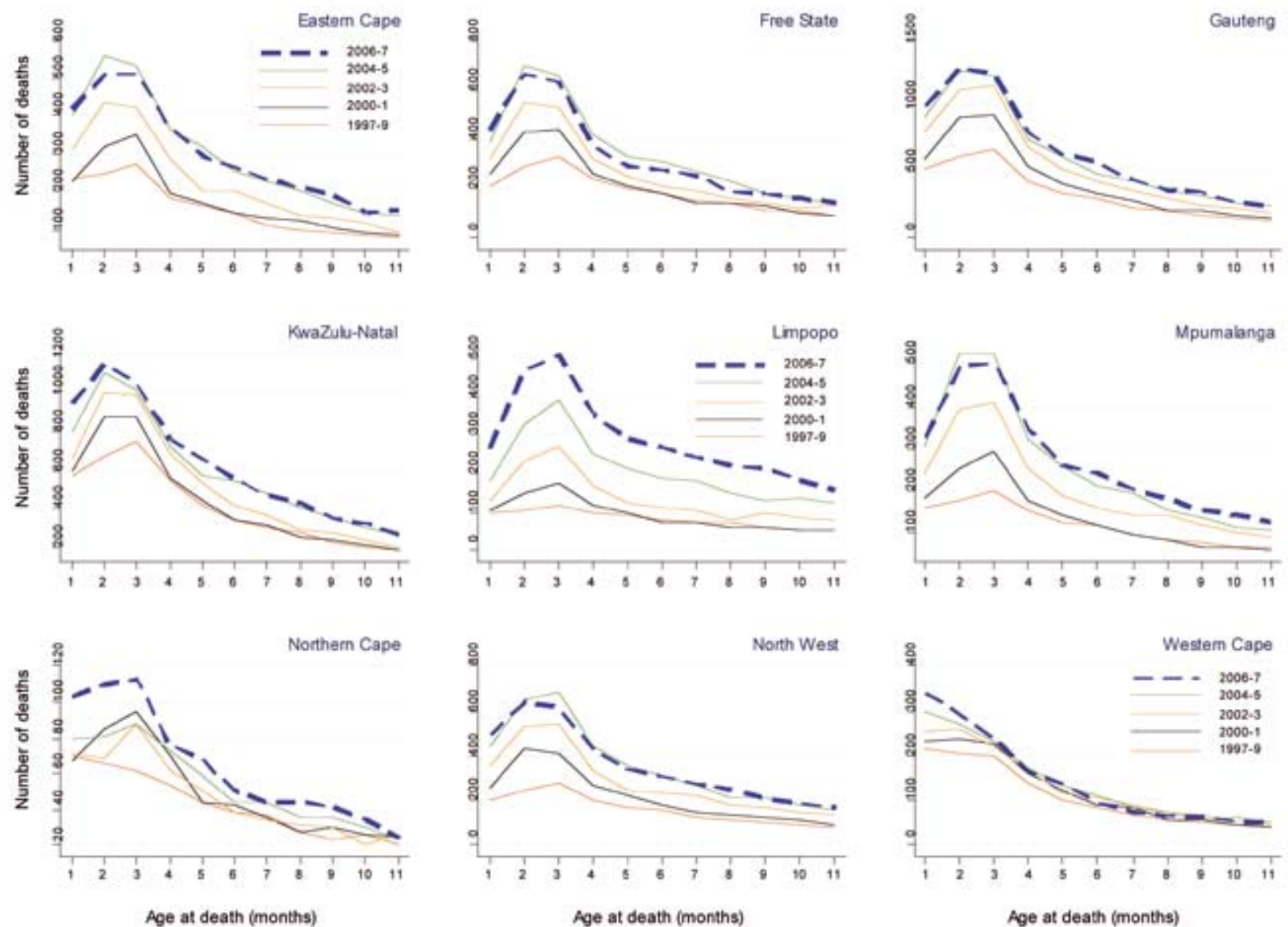

Fig. 1. Registered post-neonatal deaths in South African infants by age in months, province and year of death registration (note that the scale differs between provinces). 


\begin{tabular}{|c|c|c|c|c|c|c|c|c|c|c|c|}
\hline & & EC & FS & GT & KZN & LP & MP & $\mathrm{NC}$ & NW & wC & SA \\
\hline \multirow[t]{4}{*}{ Births $(100000 s)^{*}$} & 2001 & 1.66 & 0.64 & 2.08 & 2.48 & 1.53 & 0.85 & 0.18 & 0.85 & 1.00 & 11.27 \\
\hline & 2003 & 1.61 & 0.63 & 2.10 & 2.45 & 1.52 & 0.84 & 0.18 & 0.84 & 1.01 & 11.15 \\
\hline & 2005 & 1.59 & 0.62 & 2.04 & 2.41 & 1.53 & 0.82 & 0.18 & 0.83 & 1.00 & 11.00 \\
\hline & 2007 & 1.61 & 0.61 & 1.94 & 2.38 & 1.55 & 0.82 & 0.18 & 0.82 & 0.99 & 10.84 \\
\hline \multicolumn{12}{|l|}{ Registered infant deaths, } \\
\hline \multirow[t]{4}{*}{$1-11$ months (1 000s) } & 2001 & 1.97 & 2.43 & 5.06 & 5.09 & 1.08 & 1.60 & 0.55 & 2.59 & 1.36 & 21.72 \\
\hline & 2003 & 2.77 & 3.06 & 6.35 & 5.87 & 1.62 & 2.43 & 0.53 & 3.25 & 1.43 & 27.31 \\
\hline & 2005 & 3.47 & 3.88 & 7.09 & 7.07 & 2.50 & 3.13 & 0.51 & 4.13 & 1.55 & 33.33 \\
\hline & 2007 & 3.24 & 3.45 & 7.21 & 6.75 & 3.25 & 2.91 & 0.80 & 3.56 & 1.67 & 32.83 \\
\hline \multirow{5}{*}{$\begin{array}{l}\text { Antenatal HIV seroprevalence } \\
(\%)^{\dagger}\end{array}$} & & & & & & & & & & & \\
\hline & 2001 & 21.7 & 30.1 & 29.8 & 33.5 & 14.5 & 29.2 & 15.9 & 25.2 & 8.6 & 24.8 \\
\hline & 2003 & 27.1 & 30.1 & 29.6 & 37.5 & 17.5 & 32.6 & 16.7 & 29.9 & 13.1 & 27.9 \\
\hline & 2005 & 29.5 & 30.3 & 32.4 & 39.1 & 21.5 & 34.8 & 18.5 & 31.8 & 15.7 & 30.2 \\
\hline & 2007 & 26.0 & 33.5 & 30.3 & 37.4 & 18.5 & 32.0 & 16.1 & 29.0 & 16.1 & 28 \\
\hline \multirow{5}{*}{$\begin{array}{l}\text { HIV testing of pregnant women } \\
(\%)^{\ddagger}\end{array}$} & & & & & & & & & & & \\
\hline & 2001 & 1.7 & 4.6 & & 7.2 & 1.0 & 0.6 & 5.0 & 2.2 & & 6.9 \\
\hline & 2003 & & 31.1 & 17.6 & & 26.0 & 10.9 & 18.2 & & 86.0 & 25.3 \\
\hline & 2005 & & 40.4 & 47.4 & 43.8 & 46.5 & 31.4 & 59.1 & 47.9 & & 49.1 \\
\hline & 2007 & 75.3 & 80.1 & 73.3 & 70.7 & 90.1 & 74.6 & 88.5 & 85.6 & 95.7 & 81.0 \\
\hline \multirow[t]{4}{*}{ Adult ART coverage $(\%)^{\S}$} & 2001 & 3.0 & 2.5 & 3.9 & 3.4 & 2.2 & 2.9 & 3.3 & 2.4 & 5.1 & 3.3 \\
\hline & 2003 & 5.2 & 2.9 & 10.0 & 4.6 & 3.0 & 4.2 & 5.8 & 3.4 & 33.9 & 6.7 \\
\hline & 2005 & 26.6 & 16.6 & 37.0 & 32.4 & 24.2 & 20.5 & 42.5 & 35.8 & 66.5 & 32.8 \\
\hline & 2007 & 40.9 & 36.8 & 58.4 & 56.5 & 47.7 & 44.0 & 81.3 & 40.7 & 89.2 & 54.0 \\
\hline \multirow[t]{4}{*}{ Paediatric ART coverage $(\%)^{\S}$} & 2001 & 1.1 & 1.2 & 1.8 & 1.4 & 0.7 & 1.2 & 1.5 & 1.1 & 2.5 & 1.4 \\
\hline & 2003 & 2.3 & 1.7 & 6.9 & 2.2 & 1.2 & 1.8 & 3.9 & 1.6 & 36.8 & 3.9 \\
\hline & 2005 & 13.1 & 11.7 & 31.1 & 20.8 & 9.0 & 12.2 & 51.8 & 18.9 & 58.5 & 20.6 \\
\hline & 2007 & 26.8 & 22.1 & 46.0 & 30.6 & 35.9 & 29.4 & 96.1 & 50.7 & 96.9 & 36.9 \\
\hline \multicolumn{12}{|c|}{ 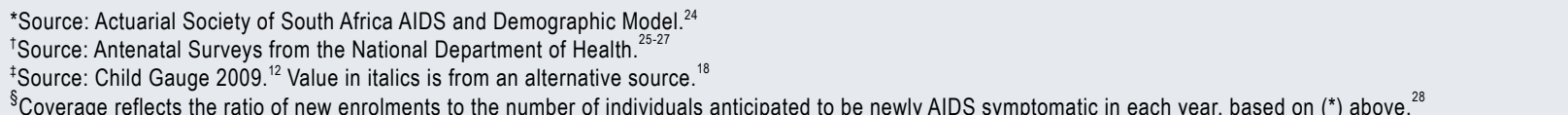 } \\
\hline
\end{tabular}

provinces in $2006-2007$. The analysis further identifies the absence of a peak in mortality at $2-3$ months in the Western Cape.

The rapid rise in registered deaths over the period 1997 - 2005 is largely attributable to improvements in the registration of infant deaths, ${ }^{13}$ but could also in part be due to increasing levels of maternal HIV prevalence (Table I), which have led to increasing levels of vertical HIV transmission and AIDS mortality. The absence of a further increase after 2005 in registered early infant deaths in 7 of the 9 provinces could potentially be ascribed to the slowing of the year-on-year increases in the completeness of infant death registration, ${ }^{13}$ the stable proportion of pregnant women with HIV since 2005, and the accelerated scale-up of prevention of mother-to-child transmission (PMTCT) and ART interventions after 2005 (Table I). The absence of this peak in the Western Cape corresponds with a delayed provincial HIV epidemic, and exceptionally high coverage of pregnant women with PMTCT interventions of increasing effectiveness.

PMTCT was first introduced in the Western Cape in 1999 in Khayelitsha ${ }^{14}$ following the protocols that had been effective in Thailand..$^{15}$ At the time this was the sub-district in the province with the highest antenatal HIV seroprevalence (22\% in 2001). ${ }^{16}$ Between 2000 and
2003, PMTCT interventions were extended to the rest of the province based on the HIVNET-012 protocols, ${ }^{17}$ reaching universal availability and over $80 \%$ coverage in early 2003. ${ }^{18}$ By early 2004 the intervention had been intensified, combining antenatal zidovudine with peripartum nevirapine, and both nevirapine and zidovudine prophylaxis to neonates. ${ }^{19,20}$ In contrast, in other provinces PMTCT was only officially sanctioned in 18 pilot sites in 2001. In 2003 less than a quarter of pregnant women in South Africa received HIV testing, and less than half in 2005 (Table I), ${ }^{12}$ with more than a third of those testing HIV positive in these provinces in 2005 not receiving even the moderately effective single-dose nevirapine-based interventions that were available at the time. ${ }^{21} \mathrm{~A}$ plausible explanation for the absence of the peak in infant deaths at 2 - 3 months of age in the Western Cape is that by the time the proportion of pregnant women who were HIV-infected reached high levels, the majority were able to access PMTCT interventions, which increased in effectiveness over time. In addition to the high coverage of PMTCT in the province, the inclusion of antenatal zidovudine in provincial PMTCT protocols probably resulted in less intra-uterine vertical transmission, which is believed to contribute relatively more to early infant mortality than intrapartum and postpartum transmission. ${ }^{9,22}$ 
A decline in under-2 mortality has recently been described in a rural KwaZulu-Natal setting after 2004, ${ }^{4}$ ascribed in part to the PMTCT programme that was rapidly scaled up in the surveillance area before the observed decline. The KwaZulu-Natal study found that the major part of this decline was in post-neonatal infant deaths, in keeping with the currently presented data.

The current study has a number of limitations. The ecological design cautions us to consider alternative explanations for our observations. As discussed, the lack of clarity on the completeness of infant death registration complicates the interpretation of these data. Further, we did not attempt to analyse mortality trends by cause of death, owing to the low proportion of HIVrelated deaths recorded as being due to HIV. ${ }^{23}$ We have, however, demonstrated previously that the peak at 2 3 months of age was absent in the small proportion of infant deaths where HIV was unlikely to be related to the underlying cause. ${ }^{8}$

We have identified a single province out of 9 South African provinces in which a peak in early infant deaths at 2 - 3 months of age did not occur during the period 1997 - 2007. This was the province with the earliest and highest coverage of ARV interventions from 1999 onwards. It is possible that these interventions have averted the greater increase in early infant deaths seen in the rest of South Africa over this period. Prospective monitoring of the age pattern of infant deaths by province could in future prove an important tool for assessing the impact of ARV and PMTCT interventions on child survival in South Africa as the completeness of infant death registration stabilises, and the coverage and effectiveness of interventions are further intensified.

We dedicate this report to the memory of our friend and colleague, David Bourne, who died unexpectedly in February 2009. David worked as a statistician and demographer in the School of Public Health and Family Medicine at the University of Cape Town for over 30 years, and was a passionate voice in describing the impact of HIV in South Africa. In recent years he became increasingly interested in the use of routine surveillance to track the evolving HIV epidemic, and in particular the age distribution of infant deaths, initiating among others this analysis, which was in process at the time of his death.

\section{REFERENCES}

1. World Health Organization. Towards Universal Access: Scaling up Priority HIVI AIDS in the Health Sector. Progress Report. Geneva: WHO, 2008.

2. Stringer JS, Zulu I, Levy J, et al. Rapid scale-up of antiretroviral therapy at primary care sites in Zambia: feasibility and early outcomes. JAMA 2006;296(7):782-793.

3. Boulle A, Bock P, Osler M, et al. Antiretroviral therapy and early mortality in South Africa. Bull World Health Organ 2008;86(9):678-687.

4. Ndirangu J, Newell ML, Tanser F, Herbst AJ, Bland R. Decline in early life mortality in a high HIV prevalence rural area of South Africa: evidence of HIV prevention or treatment impact? AIDS 2010;24(4):593-602

5. Herbst AJ, Cooke GS, Barnighausen T, KanyKany A, Tanser F, Newell ML. Adult mortality and antiretroviral treatment roll-out in rural KwaZulu-Natal, South Africa. Bull World Health Organ 2009;87(10):754-762.

6. Jahn A, Floyd S, Crampin AC, et al. Population-level effect of HIV on adult mortality and early evidence of reversal after introduction of antiretroviral therapy in Malawi. Lancet 2008;371:1603-1611.

7. Statistics South Africa. Mortality and Causes of Death in South Africa, 2006: Findings from Death Notification. Pretoria: Statistics South Africa, 2008.

8. Bourne DE, Thompson M, Brody LL, et al. Emergence of a peak in early infant mortality due to HIVIAIDS in South Africa. AIDS 2009;23(1):101-106.

9. Marinda E, Humphrey JH, lliff PJ, et al. Child mortality according to maternal and infant HIV status in Zimbabwe. Pediatr Infect Dis J 2007;26(6):519-526.

10. Newell ML, Coovadia H, Cortina-Borja M, Rollins N, Gaillard P, Dabis F. Mortality of infected and uninfected infants born to HIV-infected mothers in Africa: a pooled analysis. Lancet 2004;364:1236-1243.

11. National Department of Health. 2008 National Antenatal Sentinel HIV \& Syphilis Prevalence Survey. Pretoria: Department of Health, 2009.

12. Johnson L. Child health: HIVIAIDS. In: Pendlebury S, Lake L, Smith C, eds. South African Child Gauge 2008/2009. Cape Town: Children's Institute, University of Cape Town, 2009.

13. Darikwa TB. Estimating the level and trends of child mortality in South Africa, 19962006. MPhil (Demography) thesis, University of Cape Town, 2009.

14. Abdullah MF, Young T, Bitalo L, Coetzee N, Myers JE. Public health lessons from a pilot programme to reduce mother-to-child transmission of HIV-1 in Khayelitsha. S Afr Med J 2001:91(7):579-583.

15. Shaffer N, Chuachoowong R, Mock PA, et al. Short-course zidovudine for perinatal HIV-1 transmission in Bangkok, Thailand: a randomised controlled trial. Bangkok Collaborative Perinatal HIV Transmission Study Group. Lancet 1999;353:773-780.

16. Western Cape Department of Health. Antenatal HIV and Syphilis Prevalence Survey 2001. Cape Town: Western Cape Department of Health, 2002

17. Guay LA, Musoke $P$, Fleming $T$, et al. Intrapartum and neonatal single-dose nevirapine compared with zidovudine for prevention of mother-to-child transmission of HIV-1 in Kampala, Uganda: HIVNET 012 randomised trial. Lancet 1999;354:795802.

18. Draper B, Abdullah F. A review of the prevention of mother-to-child transmission programme of the Western Cape provincial government, 2003 - 2004. S Afr Med J 2008;98(6):431-434

19. Lallemant M, Jourdain G, Le Coeur S, et al. Single-dose perinatal nevirapine plus standard zidovudine to prevent mother-to-child transmission of HIV-1 in Thailand. N Engl J Med 2004:351(3):217-228.

20. Coetzee D, Hilderbrand K, Boulle A, Draper B, Abdullah F, Goemaere E. Effectiveness of the first district-wide programme for the prevention of mother-tochild transmission of HIV in South Africa. Bull World Health Organ 2005;83(7):489494.

21. Health Systems Trust. District Health Barometer 2007/08. Durban: Health Systems Trust, 2009.

22. Mayaux MJ, Burgard M, Teglas JP, et al. Neonatal characteristics in rapidly progressive perinatally acquired HIV-1 disease. The French Pediatric HIV Infection Study Group. JAMA 1996:275(8):606-610.

23. Yudkin PL, Burger EH, Bradshaw D, Groenewald P, Ward AM, Volmink J. Deaths caused by HIV disease under-reported in South Africa. AIDS 2009;23(12):16001602

24. ASSA2003lite Aids and Demographic Model of the Acturial Society of South Africa. http://www.assa.org.za (accessed 7 May 2009).

25. National Department of Health. The National HIV and Syphilis Prevalence Survey South Africa 2003. Pretoria: Department of Health, 2004.

26. National Department of Health. The National HIV and Syphilis Prevalence Survey South Africa 2005. Pretoria: Department of Health, 2006.

27. National Department of Health. The National HIV and Syphilis Prevalence Survey South Africa 2007. Pretoria: Department of Health, 2008.

28. Johnson L. HIV and health - access to PMTCT: HIV testing. Children count: Statistics on children in South Africa. Cape Town: Children's Institute, University of Cape Town, 2009. http://www.childrencount.ci.org.za (accessed 24 May 2010). 